\title{
Robust Adaptive Control of PVTOL System via Minimum Projection Method
}

\author{
Soki Kuga ${ }^{1}$, Hisakazu Nakamura ${ }^{1}$ and Yasuyuki Satoh ${ }^{2}$ \\ 1. Tokyo University of Science, Yamazaki 2641, Noda, Chiba 278-8150, Japan (e-mail: sokijournal@gmail.com) \\ 2. Kyoto University (yasuyuki.satoh@gmail.com)
}

Received: November 12, 2015 / Accepted: December 15, 2015 / Published: May 25, 2016.

\begin{abstract}
Dynamic extension" is commonly used for stabilization of the planar vertical take off and landing (PVTOL) system. Most controllers designed by the method are based on "dynamic" control Lyapunov functions (CLFs). We design a $C^{\infty}$ differentiable "static" CLF for the PVTOL system by dynamic extension and minimum projection method. Then we propose an inverse optimal controller based on the static CLF that attains a gain margin. We design an adaptive control input and show the robustness of the controller by computer simulation.
\end{abstract}

Key words: Nonlinear control, Control Lyapunov function, Adaptive control, Inverse optimal control, PVTOL system.

\section{Introduction}

VTOL aircrafts such as V-22 or quadrotors attract much attentions in recent years. The planar vertical take off and landing (PVTOL) system introduced by Hauser et al. [1] is a useful model to study position control of VTOL aircrafts.

A common and useful control strategy is one of proposed by Hauser, based on dynamic extension. Researchers proposed many controllers based on dynamic extension [1]-[5]. The method stabilizes an arbitrary operating point with a large domain of attraction of the state space.

However, these controllers are based on smooth "dynamic" control Lyapunov functions (CLFs), and lost robustness due to their dynamic input transformations [1] [3] [6]. Moreover, a static CLF-based control strategy can not beapplied.

In this paper, we propose a $C^{\infty}$ differentiable "static" CLF for stabilization of the PVTOL system. The proposed CLF is designed by the following two

Corresponding author: Soki Kuga, Tokyo University of Science, Yamazaki 2641, Noda, Chiba 278-8150, Japan. E-mail: sokijournal@gmail.com. steps. First, we design a "dynamic" CLF for the augmented system of the PVTOL system obtained by dynamic extension. Then we design a static CLF for the PVTOL system by minimum projection method proposed by Yamazaki et al. [7]

Our proposed CLF is a smooth function. We propose an adaptive control law with an inverse optimal controller based on the designed smooth static CLF. Finally, we show computer simulation to confirm that our controller can stabilize the origin of the PVTOL system under parameter uncertainty.

\section{Preliminaries}

\subsection{Control System on Extended Space}

Consider the following nonlinear control system:

$$
\dot{x}=f(x)+g(x) u,
$$

where $x \in \mathbb{R}^{n}$ and $u \in \mathbb{R}^{m}$ denote a state and an input respectively. Mappings $f: \mathbb{R}^{n} \rightarrow \mathbb{R}^{n}$ and $g: \mathbb{R}^{n} \rightarrow \mathbb{R}^{n \times m}$ are supposed to be Lipschitz continuous with respect to both $x$ and $u$, and satisfy $f(0,0)=0 \quad$ [8]. 
This paper also considers the following augmented system of (1) on extended space $\mathbb{R}^{n+l}$ :

$$
\begin{gathered}
\dot{\tilde{x}}=\left[\begin{array}{c}
\dot{x}(2) \\
\dot{p}
\end{array}\right]=\left[\begin{array}{c}
f(x)+g(x) u(3) \\
v
\end{array}\right] \\
=\tilde{f}(x)+\tilde{g}(x) \tilde{u},
\end{gathered}
$$

where $p \in \mathbb{R}^{l}$ denotes a virtual state, $v \in \mathbb{R}^{l}$ a virtual input and $\tilde{u}=[u, v]^{T}$.

\subsection{Control Lyapunov Function (CLF)}

In this paper, we design a controller based on differentiable control Lyapunov functions (CLFs). Throughout this paper, state spaces $X, X, \tilde{X}$ and $\bar{X}$ are supposed to be neighborhoods of the origin.

Definition 1. Control Lyapunov function $(C L F)$ [10] Consider system (1). Then a proper positive definite $C^{\infty}$ differentiable function $V: \mathbb{R}^{n} \supset X$ $\rightarrow \mathbb{R}$ satisfying the following condition is said to be a $C^{\infty}$ control Lyapunov function (CLF) for (1):

$$
L_{f} V(x)<0, \forall x \in\left\{x \in X \mid L_{g} V=0\right\},
$$

where $\quad L_{f} V=(\partial V / \partial x) f(x)$ and $L_{g} V=$ $(\partial V / \partial x) g(x)$. Then we define $\dot{V}(x, u)$ as follows:

$$
\dot{V}(x, u)=L_{f} V(x)+L_{g} V(u) .
$$

Remark 1. Yamazaki et al. [7] introduces Sontag's CLF as the definition of the obtained CLF by minimum projection method. In this paper, we use stronger CLF definition denoted above such as Artstein [11] or Nakamura et al. [10].

Freeman [9] proposed a CLF design method for a nonlinear control system. For a feedback linearizable system, the following proposition holds:

Proposition 1. Suppose there exists a diffeomorphism $\varphi=\Phi(x)$ with $\Phi(0)=0$ which transforms system (1) into

$$
\dot{\varphi}=A \varphi+B\left[l_{0}(\varphi)+l_{1}(\varphi) u\right],
$$

where the matrix pair $(A, B)$ is controllable and mappings $l_{0}: \mathbb{R}^{n} \rightarrow \mathbb{R}^{n}$ and $l_{1}: \mathbb{R}^{n} \rightarrow \mathbb{R}^{n \times m}$ are continuous, and $l_{1}(\Phi)$ is nonsingular for all $\Phi$.

Let $P$ be a symmetric positive definite solution of the following Riccati equation:

$$
A^{T} P+P A-P B R^{-1} B^{T} P+Q=0
$$

for arbitrary positive definite matrices $Q$ and $R$.

Then function $\tilde{V}(x): \mathbb{R}^{n} \supset X \rightarrow \mathbb{R}$ defined by the following function is a CLF for (1):

$$
\tilde{V}(x)=\Phi^{T}(x) P \Phi(x) .
$$

\subsection{CLF Design via Dynamic Extension}

Yamazaki et al. [7] proposed a nonsmooth CLF design method via minimum projection method. A CLF for the system (1) is heavily related to a CLF for the augmented system (2) as shown in the following theorem.

Theorem 1. Let a continuous function $\bar{V}: \mathbb{R}^{n+l} \supset \bar{X} \rightarrow \mathbb{R}$ be a CLF for (2). Then the following function $V: \mathbb{R}^{n} \supset X \rightarrow \mathbb{R}$ is a weak CLF for (1):

$$
V(x)=\min _{p \in \mathbb{R}^{l}} \bar{V}(x, p) .
$$

Note that Theorem 1 does not guarantee the differentiability of $V$.

\subsection{Inverse Optimal Control with CLF}

Nakamura et al. [10] proposed an inverse optimal controller with a CLF. One of advantages of the inverse optimal control is a gain margin for robustness of the controllers defined below.

Definition 2. Gain margin Consider a state feedback control $u=k(x)$. The control input $u \in \mathbb{R}^{m}$ for system (1) is said to have a gain margin $[\alpha, \beta)$ if the origin of the closed-loop system $\dot{x}=f(x)+g(x) \Delta u$ is asymptotically stable for any $\Delta \in \mathbb{R}^{m \times m}$ which is of the form diag $\left\{\kappa_{i}, \ldots, \kappa_{m}\right\}$ with constants $\kappa_{i} \in[\alpha, \beta), i=1, \ldots, m$. 
With a CLF, we can design an inverse optimal controller achieving a gain margin by the following lemma:

Lemma 1. [10] Let $a_{j}>0$ be a constant for all $j \in\{1, \ldots, m\}, \quad V: \mathbb{R}^{n} \supset X \rightarrow \mathbb{R} \quad$ a CLF for system (1). Then we define $\gamma$ as follows:

$$
\gamma(x)=\sum_{j=1}^{m} \frac{1}{a_{j}+1} \cdot \frac{1}{R(x)}\left|L_{g_{j}} V(x)\right|^{a_{j}+1}-L_{f} V(x),
$$

where $R: X \rightarrow \mathbb{R}_{>0}$ is a positive-valued function on $X \backslash\{0\}$ and $\gamma(x)$ is a positive definite function. Then the following input $u_{j}: \mathbb{R}^{n} \supset X \rightarrow \mathbb{R}^{m}$ asymptotically stabilizes the origin of the system (1):

$$
\begin{gathered}
u_{j}(x)=-\frac{1}{R(x)}\left|L_{g_{j}} V(x)\right|^{a_{j}} \operatorname{sgn}\left(L_{g_{j}} V(x)\right), \\
(j=1, \ldots, m),
\end{gathered}
$$

and minimizes the following cost functional:

$$
J=\int_{0}^{\infty}\left[\gamma(x)+\sum_{j=1}^{m} \frac{a_{j}}{a_{j}+1} R^{1 / a_{j}}(x)\left|u_{j}\right|^{\left(a_{j}+1\right) / a_{j}}\right] d t .
$$

Further the input (11) is continuous on $X \backslash\{0\}$ and achieves at least a gain margin $\left[1 /\left(\min _{1 \leq j \leq m} a_{j}+1\right), \infty\right)$.

\section{Problem Statement}

The following equation denotes a normalized equation of motion of the PVTOL aircraft with gravitational acceleration $g=1$ introduced by Hauser[1] (Fig. 1):

$$
\dot{x}=\left[\dot{x}_{1}, \dot{x}_{2}, \dot{x}_{3}, \dot{x}_{4}, \dot{x}_{5}, \dot{x}_{6}\right]^{T}
$$

$$
=\left[\begin{array}{c}
x_{2} \\
0 \\
x_{4} \\
-1 \\
x_{6} \\
0
\end{array}\right]+\left[\begin{array}{cc}
0 & 0 \\
-\sin x_{5} & \varepsilon \cos x_{5} \\
0 & 0 \\
\cos x_{5} & \varepsilon \sin x_{5} \\
0 & 0 \\
0 & 1
\end{array}\right]\left[\begin{array}{l}
u_{1} \\
u_{2}
\end{array}\right],
$$

where $x=\left[x_{1}, x_{2}, \ldots, x_{6}\right]^{T} \in \mathbb{R}^{6}$ and $u=\left[u_{1}, u_{2}\right]^{T}$ $\in R^{2}$ denote respective a state variable and a control input. $u_{1}, u_{2}$ are the vertical thrust attached to the aircraft and rolling moment respectively. The normalized lengths $x_{1}, x_{3}$ represent actual lengths divides by gravitational acceleration $g\left(\mathrm{~m} / \mathrm{s}^{2}\right)$; a normalized length of 1 represents an actual length of about $10 \mathrm{~m}$ [13].

$\varepsilon$ denotes a coupling coefficient between $u_{2}$ and lateral acceleration $\varepsilon u_{2}$. When small $\varepsilon$, the system is a strong non-minimum phase system. In many VTOL aircrafts, thrust spindles are vertically attached to the body; i.e., the inputs coupling $\varepsilon=0$ structurally. Hence in this paper we suppose $\varepsilon=0$; the system becomes an under-actuated system.

" -1 " appeared in both Fig. 1 and the first term of the right-hand side of (13) denotes the normalized gravitational acceleration. Note that the origin of (13)

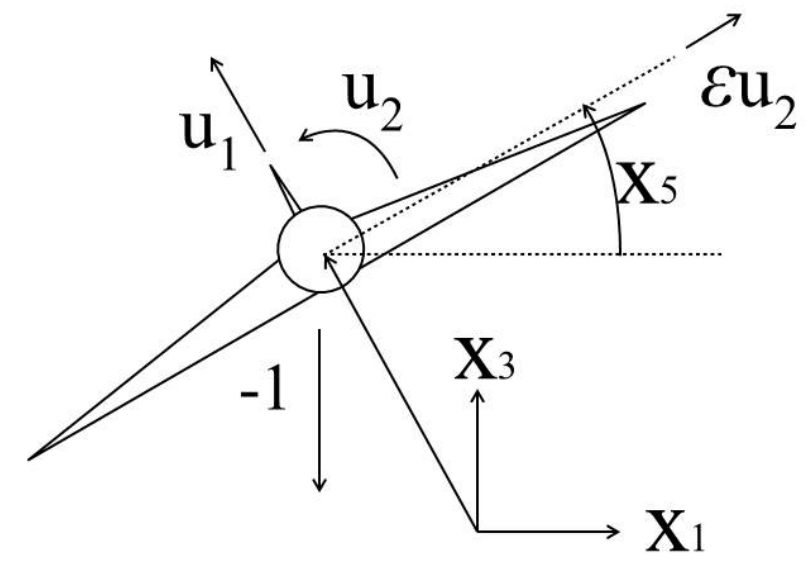

Fig. 1 The PVTOL aircraft. 
is an equilibrium when $u_{1}=1$. Let a new vertical control input $\tilde{u}_{1}=u_{1}-1$, and then we obtain the following control system:

$$
\begin{gathered}
\dot{x}=\left[\begin{array}{c}
x_{2} \\
-\sin x_{5} \\
x_{4} \\
\cos x_{5}-1 \\
x_{6} \\
0
\end{array}\right]+\left[\begin{array}{cc}
0 & 0 \\
-\sin x_{5} & 0 \\
0 & 0 \\
\cos x_{5} & 0 \\
0 & 0 \\
0 & 1
\end{array}\right]\left[\begin{array}{l}
\tilde{u}_{1} \\
u_{2}
\end{array}\right] \\
=f(x)+g_{1}(x) \tilde{u}_{1}+g_{2}(x) u_{2} .
\end{gathered}
$$

In this paper, we consider a static state feedback controller design problem for asymptotic stabilization of the origin of (14).

\section{Conventional Dynamic Extension to PVTOL System}

In this section, we apply the conventional dynamic extension to PVTOL system (14), and obtain a dynamic CLF for the linear augmented system of (14).

In accordance with Hauser's dynamic extension, we consider the following coordinate and input transformation with a new state variable $\varphi \in \tilde{X} \subset \mathbb{R}^{8}$ and $v \in \mathbb{R}^{2}$ :

$$
\begin{aligned}
& \varphi=\left[\varphi_{1}, \varphi_{2}, \ldots, \varphi_{8}\right]^{T}=\Phi\left(x, \tilde{u}_{1}, \dot{\tilde{u}}_{1}\right) \\
& {\left[\begin{array}{c}
x_{1} \\
x_{2} \\
-\left(1+\tilde{u}_{1}\right) \sin x_{5} \\
-\left(1+\tilde{u}_{1}\right) x_{6} \cos x_{5}-\dot{\tilde{u}}_{1} \sin x_{5} \\
x_{3} \\
x_{4} \\
\left(1+\tilde{u}_{1}\right) \cos x_{5}-1 \\
-\left(1+\tilde{u}_{1}\right) x_{6} \sin x_{5}+\dot{\tilde{u}}_{1} \cos x_{5}
\end{array}\right]} \\
& v=\left[\begin{array}{l}
v_{1} \\
v_{2}
\end{array}\right]
\end{aligned}
$$

$$
\begin{aligned}
& =\left[\begin{array}{cc}
x_{6}^{2} \sin x_{5} & -2 x_{6} \cos x_{5} \\
-x_{6}^{2} \cos x_{5} & -2 x_{6} \sin x_{5}
\end{array}\right]\left[\begin{array}{c}
\tilde{u}_{1}+1 \\
\dot{\tilde{u}}_{1}
\end{array}\right] \\
& +\left[\begin{array}{cc}
-\sin x_{5} & -\left(\tilde{u}_{1}+1\right) \cos x_{5} \\
\cos x_{5} & -\left(\tilde{u}_{1}+1\right) \sin x_{5}
\end{array}\right]\left[\begin{array}{c}
\ddot{u}_{1} \\
u_{2}
\end{array}\right],
\end{aligned}
$$

Then we can transform the system (1) into the following linear augmented control system:

$$
\dot{\varphi}=\left[\varphi_{2}, \varphi_{3}, \varphi_{4}, v_{1}, \varphi_{6}, \varphi_{7}, \varphi_{8}, v_{2}\right]^{T} .
$$

We can easily design a dynamic CLF $\tilde{V}: \mathbb{R}^{8} \supset \tilde{X} \rightarrow \mathbb{R}$ for the linear augmented system (18) as follows:

$$
\tilde{V}(\varphi)=\varphi^{T} P_{0} \varphi,
$$

where $P_{0}$ is a solution of the Riccati equation denoted in Proposition 1 for (18) with $R=I$ and $Q=I$.

According to Proposition 1, the following function $\bar{V}: \mathbb{R}^{8} \supset \bar{X} \rightarrow \mathbb{R}$ is a CLF for (14) with respect to $\Phi\left(x, \tilde{u}_{1}, \dot{\tilde{u}}_{1}\right) \subset \mathbb{R}^{8}:$

$$
\begin{aligned}
& \bar{V}\left(x, \tilde{u}_{1}, \dot{\tilde{u}}_{1}\right):=\tilde{V}\left(\Phi\left(x, \tilde{u}_{1}, \dot{\tilde{u}}_{1}\right)\right) \\
& =\Phi^{T}\left(x, \tilde{u}_{1}, \dot{\tilde{u}}_{1}\right) P_{0} \Phi\left(x, \tilde{u}_{1}, \dot{\tilde{u}}_{1}\right) .
\end{aligned}
$$

Remark 2. Note that the input transformation (17) is valid locally in the neighborhood of the operating point $x_{1}, x_{3}=0$ due to the following inverse mapping of (17) has a singularity at $\tilde{u}_{1}=-1$ :

$$
\begin{gathered}
{\left[\begin{array}{c}
\ddot{\tilde{u}}_{1} \\
u_{2}
\end{array}\right]=\left[\begin{array}{c}
x_{6}^{2}\left(\tilde{u}_{1}+1\right) \\
-\frac{2 x_{6} \dot{\tilde{u}}_{1}}{\left(\tilde{u}_{1}+1\right)}
\end{array}\right]} \\
+\left[\begin{array}{ll}
-\sin x_{5} & \cos x_{5} \\
\frac{-\cos x_{5}}{\left(\tilde{u}_{1}+1\right)} & \frac{\sin x_{5}}{\left(\tilde{u}_{1}+1\right)}
\end{array}\right]\left[\begin{array}{l}
v_{1} \\
v_{2}
\end{array}\right] .
\end{gathered}
$$

This singularity is due to dynamic input transformationis introduced in [6]. Here, $\bar{V}$ is valid in the following set due to the singularity: 


$$
\bar{X}=\left\{\left(x, \tilde{u}_{1}, \dot{\tilde{u}}_{1}\right) \mid \bar{V}\left(x, \tilde{u}_{1}, \dot{\tilde{u}}_{1}\right)<\bar{R}_{\max }\right\},
$$

where $\bar{R}_{\max }=\min _{\tilde{u}_{1}=-1} \bar{V}\left(x, \tilde{u}_{1}, \dot{\tilde{u}}_{1}\right)=2.44$.

Remark 3. Dynamic control input $v$ designed by dynamic extension may lost robustness due to the dynamic input transformation (22).

\section{Minimum Projection Method to PVTOL System}

\subsection{Static CLF Design for PVTOL system}

In this section, we develop a $C^{\infty}$ differentiable "static" CLF for the PVTOL system (14) based on minimum projection method Theorem 1 proposed by Yamazaki et al. [7]

Theorem 2. Let the function $\bar{V}: \mathbb{R}^{8} \supset \bar{X} \rightarrow \mathbb{R}$ denoted in (21) be a $C^{\infty}$ differentiable CLF for system (18). Then the following function $V: \mathbb{R}^{6} \supset X \rightarrow \mathbb{R}$ is a $C^{\infty}$ differentiable CLF for system (1):

$$
\begin{aligned}
V(x)= & \Phi^{T}\left(x, p_{1}(x), p_{2}(x)\right) P_{0} \Phi\left(x, p_{1}(x), p_{2}(x)\right) \\
& =\Phi^{T}(x, p(x)) P_{0} \Phi(x, p(x)),
\end{aligned}
$$

where the function $p(x)=\left[p_{1}, p_{2}\right]: \mathbb{R}^{6} \rightarrow \mathbb{R}^{2}$ is uniquely determined by the folloing equations:

$$
\begin{aligned}
& \frac{\partial \bar{V}}{\partial p_{1}}\left(x, p_{1}, p_{2}\right)=2 \Phi^{T} P_{0} \frac{\partial \Phi}{\partial p_{1}}=0, \\
& \frac{\partial \bar{V}}{\partial p_{2}}\left(x, p_{1}, p_{2}\right)=2 \Phi^{T} P_{0} \frac{\partial \Phi}{\partial p_{2}}=0
\end{aligned}
$$

that minimizes $\bar{V}$ with respect to $\tilde{u}_{1}$ and $\dot{\tilde{u}}_{1}$.

Note that Theorem 2 guarantees differentiability of $V$, though Theorem 1 does not consider the differentiability of the CLF obtained by minimum projection method.

In order to proof Theorem 2, we employ the following three lemmas.

Lemma 2. There exist mappings $C: \mathbb{R}^{6} \rightarrow \mathbb{R}^{2 \times 2}$, $D: \mathbb{R}^{6} \rightarrow \mathbb{R}^{2}$ that (25) is equivalent to the following equation:

$$
C(x) p=D(x) .
$$

Proof 1. Note (16), and we permit derivatives $\left(\partial \bar{V} / \partial \tilde{u}_{1}\right)\left(x, \tilde{u}_{1}, \dot{\tilde{u}}_{1}\right)$ and

$\left(\partial \bar{V} / \partial \dot{\tilde{u}}_{1}\right)\left(x, \tilde{u}_{1}, \dot{\tilde{u}}_{1}\right)$ consist of linear functions with respect to both $\tilde{u}_{1}$ and $\dot{\tilde{u}}_{1}$ for every fixed $x$.

Lemma 3. The implicit function $p=\left[p_{1}, p_{2}\right]$ : $\mathbb{R}^{6} \rightarrow \mathbb{R}^{2}$ defined by (25) is uniquely determined.

Proof 2. Note that $\bar{V}$ is proper and bounded below, and there must exist $p$ minimizing $\bar{V}$ for a fixed $x$. Properness of $\bar{V}$ guarantees that a set $L(x)=\operatorname{argmin} \bar{V}(x, p)$ is compact. This implies that the matrix $C(x)$ is nonsingular for all $x$. Therefore there exists a unique minimizer $p$ of $\bar{V}$.

Owing to nonsingularity of $C(x)$, smoothness of function $V$ is confirmed by implicit function theorem [12].

Lemma 4. The following function $V: \mathbb{R}^{6} \supset X \rightarrow \mathbb{R}$ is a $C^{\infty}$ differentiable function:

$$
\begin{gathered}
V(x)=\Phi^{T}\left(x, p_{1}(x), p_{2}(x)\right) P_{0} \Phi\left(x, p_{1}(x), p_{2}(x)\right) \\
=\Phi^{T}(x, p(x)) P_{0} \Phi(x, p(x)) .
\end{gathered}
$$

Proof 3. We consider the following mapping $F$ with mappings $C$ and $D$ in (26):

$$
F(x, p)=C(x) p-D(x) .
$$

Note that

$$
\frac{\partial F}{\partial p}=C(x)
$$

is nonsingular in $x$. Therefore, according to implicit function theorem, there exist a $C^{\infty}$ differentiable mapping $p(x)$ satisfying

$$
F(x, p(x))=0 .
$$

Then the following function

$$
V(x)=\Phi^{T}(x, p(x)) P_{0} \Phi(x, p(x))
$$

is a $C^{\infty}$ differentiable function. 


\section{Proof of Theorem 2.}

Proof 4. Let $\bar{x}=(x, p(x))$ with $p(x)$ in Lemma $3, \quad \bar{u}_{0}=\left[u_{0}, v_{0}\right]^{T}$ be constants satisfying $\dot{\bar{V}}\left(\bar{x}, u_{0}, v_{0}\right)<0$. Then the following inequality holds for sufficiently small $\delta>0$ :

$$
V\left(\psi\left(\delta t, x ; u_{0}\right)\right) \leq \bar{V}\left(\bar{\psi}\left(\delta t, \bar{x} ; \bar{u}_{0}\right)\right)<\bar{V}(\bar{x}),
$$

where $\psi(t, x ; u)$ is a unique solution of the differential equation (14) with Lebesgue measurable input $u(t) \in \mathbb{R}^{2}$ for $t$ starting at $x$, $\left.\bar{\psi}\left(\delta t, \bar{x} ; \bar{u}_{0}\right)\right)$ is also a unique solution of the differential equation (18) with Lebesgue measurable input $\bar{u}(t) \in \mathbb{R}^{4}$ for $t$ starting at $\bar{x}$ [8]. Therefore, $\dot{V}\left(x, u_{0}\right)<0$. This implies the function $V$ is a CLF in $X \subset \mathbb{R}^{6}$.

\section{Remark 4.}

The static CLF $V$ obtained by Theorem 2 is valid in the following subset:

$$
X=\left\{x \mid V(x)<R_{\max }\right\},
$$

where

$$
R_{\text {max }}=\min _{x}\left\{V(x) \mid L_{g} V(x)=0 \text { and } L_{f} V(x) \geq 0\right\} .
$$
Though it is difficult to calculate the strict value of $R_{\max }$, we can estimate the conservative value of $\mathrm{c}$. The function $V$ is always a CLF, if the input transformation (17) has its inverse mapping; (17) is nonsingular for all $x$. Conversely if (17) is singular for some $x, V$ may not be a CLF. When $\tilde{u}_{1}=-1$, (17) becomes singular. Then we can calculate the conservative value of $R_{\max }$ as follows:

$$
V(x)<\min _{p_{1}(x)=-1} V(x)=3.57 .
$$

In accordance with Lemma 9 in [15], the equality $\{x \mid V(x)<2.44\}=\{x \in \bar{X} \mid \bar{V}(x, p)<2.44\}$ is satisfied. Therefore,

$$
\{x \mid V(x)<3.57\} \supset\{x \in \bar{X} \mid \bar{V}(x, p)<2.44\}
$$

is also satisfied. Equation (32) denotes that the conservative value of the domain of attraction $V$ is at least larger than that of $\bar{V}$.

In domain of attraction $\bar{V}$, we proposed a new CLF for the PVTOL system:

Theorem 3. The following function $V: \mathbb{R}^{6} \supset X \rightarrow \mathbb{R}$ is a static CLF;

$$
V(x)=\frac{V(x) R_{\max }}{R_{\max }-V(x)} .
$$

In Fig. 2, we illustrate $V$ on the surface $x_{1}=1, x_{2}=x_{4}=x_{6}=0$.

Remark 5. Our proposed CLF $V$ and $V$ are smooth static functions. This means that many control strategy based on smooth CLFs are available.

\subsection{Static State Feedback Controller Design for PVTOL System}

We design an inverse optimal controller for PVTOL system (14) with the smooth static CLF $V$ by Lemma 1 as follows:

Proposition 2. Consider (14) and CLF $V$ designed by Theorem 3 . Then the following controller $u=k(x): \mathbb{R}^{6} \supset X \rightarrow \mathbb{R}^{2}$ asymptotically stabilizes the origin of the PVTOL system (14) and guarantees a gain margin $(1 / 2,+\infty)$ :

$$
\begin{gathered}
k(x)=\left[\begin{array}{l}
k_{1}(x) \\
k_{2}(x)
\end{array}\right]= \\
\begin{cases}-\left(\frac{L_{f} V+\left|L_{f} V\right|}{\left(L_{g_{1}} V\right)^{2}+\left(L_{g_{2}} V\right)^{2}}+C\right)\left[\begin{array}{l}
L_{g_{1}} V \\
L_{g_{2}} V
\end{array}\right] & \left(L_{g} V \neq 0\right) \\
0 & \left(L_{g} V=0\right)\end{cases}
\end{gathered}
$$

where

$$
L_{g_{1}} V=(\partial V / \partial x) g_{1}(x), L_{g_{2}} V=(\partial V / \partial x) g_{2}(x)
$$

and $C$ is an arbitrary positive constant number.

Proof 5. For all $x \in X, L_{f} V<0$ if $L_{g} V=0$. Let $m=2, a_{1}, a_{2}=1$ and 


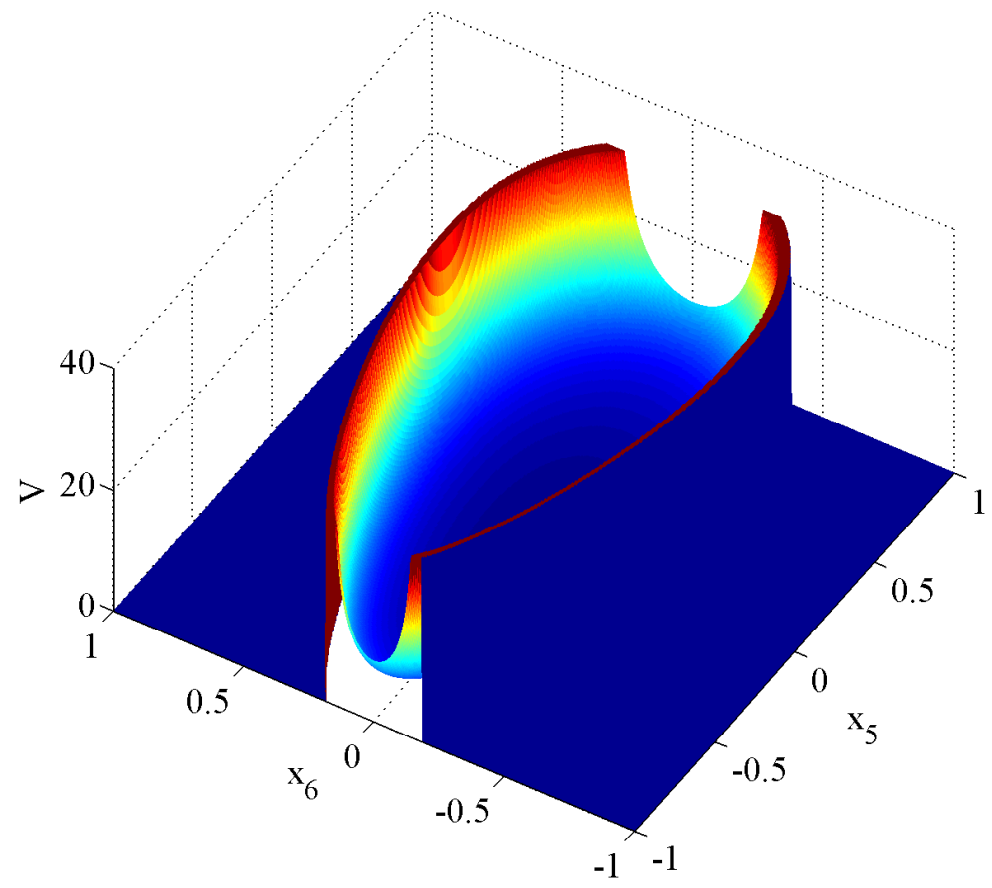

Fig. 2 The obtained CLF of the PVTOL aircraft system on $x_{1}=1, x_{2}=x_{4}=x_{6}=0$.

$$
R(x)=\frac{\left(L_{g_{1}} V\right)^{2}+\left(L_{g_{2}} V\right)^{2}}{L_{f} V+\left|L_{f} V\right|+C\left\{\left(L_{g_{1}} V\right)^{2}+\left(L_{g_{2}} V\right)^{2}\right\}} .
$$

By (10) and (11), the following inequality holds:

$$
\begin{gathered}
\gamma(x) \leq-\dot{V}\left(x, 1 /\left(\min _{1 \leq j \leq 2} a_{j}+1\right) \cdot k(x)\right) \\
=-\dot{V}\left(x, \frac{1}{2} k(x)\right) .
\end{gathered}
$$

Let each $\Delta$ be any uncertainty in $[1 / 2, \infty)$. Then by (11)

$$
\begin{gathered}
\sum_{j=1}^{m} L_{g_{j}} V(x) \cdot \Delta \cdot k_{j}(x) \\
\leq \sum_{j=1}^{2} L_{g_{j}} V(x) \cdot \frac{1}{2} k_{j}(x), \\
\forall x \in X .
\end{gathered}
$$

Hence

$$
\begin{gathered}
\dot{V}\left(x, \Delta \cdot k_{j}(x)\right) \leq \dot{V}\left(x, \frac{1}{2} k_{j}(x)\right) \leq-\gamma(x)<0, \\
\forall x \in X .
\end{gathered}
$$

Then $\dot{V}(x, u)<0, \quad \forall x \in X, \forall \Delta \in[1 / 2, \infty)$.
Hence, all assumptions in Lemma 1 hold for an arbitrary $C>0$. Therefore, the input (34) asymptotically stabilizes the origin of the system (14) for all $x \in X$, and guarantees the gain margin $[1 / 2,+\infty)$.

Remark 6. Controller (34) designed with our proposed CLF $V$ do not suffer from singularity depicted in Remark 2 because the controller do not use dynamic input transformation (22)

\section{Robustness of the Obtained Controller for the PVTOL System}

The proposed inverse optimal control input has the gain margin. This means that we can design an adaptive controller that can stabilize the origin of the PVTOL system under parameter uncertainty.

\subsection{Adaptive Control of PVTOL system}

In this section, we consider the following system which considers parameter uncertainty $w$ in the PVTOL system (14):

$$
\dot{x}=f(x)+g_{1}(x)\left(\tilde{u}_{1}+w\right)+g_{2}(x) u_{2},
$$


where the origin of the system is $x=0$. We propose a controller for asymptotic stabilization of the origin of (39).

In the system (39), parameter uncertainty $w$ occurs offset error in equilibrium point.

Fig. 3 illustrates state response of the system (39) with $w=0.1$ and a controller designed in (34) for the nominal PVTOL system (14). We can confirm a offset error due to the parameter uncertainty $w$.

We propose an adaptive control law with the smooth CLF $V$ that stabilizes the origin of the system (39).

Theorem 4. Consider system (39). We chose a controller $u$ as follows and the input asymptotically stabilizes the origin of the system (39) for all $x \in X$ :

$$
\begin{gathered}
u=\left[\begin{array}{c}
\tilde{u}_{1} \\
u_{2}
\end{array}\right]=\left[\begin{array}{c}
k_{1}(x)-\hat{w} \\
k_{2}(x)
\end{array}\right] \\
\dot{\hat{w}}=\operatorname{Lg}_{1} V(x),
\end{gathered}
$$

where V is a smooth CLF for the PVTOL system (14) designed by Theorem $3, k_{1}(x)$ and $k_{2}(x)$ are control inputs for the nominal PVTOL system (14) designed by Proposition 2, $\hat{w}$ is estimated value of $w$.

Proof 6. Consider the following proper positive definite function $\hat{V}: X \times \mathbb{R} \rightarrow \mathbb{R}_{\geq 0}$ :

$$
\hat{V}(x, \hat{w})=V(x)+\frac{1}{2}(w-\hat{w})^{2} .
$$

Here, the following inequality holds:

$$
\begin{gathered}
\dot{\hat{V}}(x, \hat{w})=\dot{V}(x)-\dot{\hat{w}}(w-\hat{w}) \\
=\frac{\partial V}{\partial x}\left[f(x)+g_{1}(x)\left(\tilde{u}_{1}+w\right)+g_{2}(x) u_{2}\right] \\
-\dot{\hat{w}}(w-\hat{w}) \\
=L_{f} V+L_{g_{1}} V \times\left(\tilde{u}_{1}+w\right) \\
+L_{g_{2}} V \times u_{2}-\dot{\hat{w}}(w-\hat{w}) \\
=L_{f} V+L_{g_{1}} V \times\left(k_{1}(x)+w-\hat{w}\right) \\
+L_{g_{2}} V \times k_{2}(x)-L_{g_{1}} V \times(w-\hat{w}) \\
=L_{f} V+L_{g_{1}} V \times k_{1}(x)+L_{g_{2}} V \times k_{2}(x) \\
\leq 0 .
\end{gathered}
$$

Then the function $\hat{V}$ is a weak Lyapunov function for the system (39). Therefore, the controller stabilize

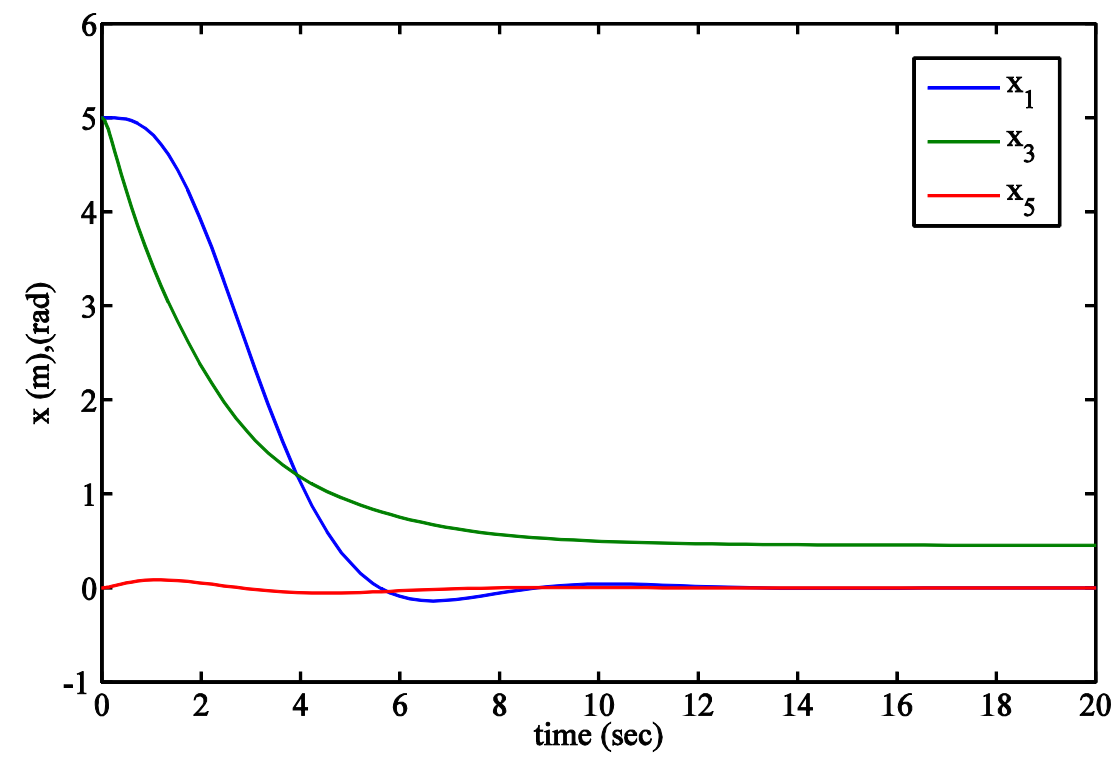

Fig. 3 State response, $x(0)=(0.5,0,0.5,0,0,0)$ under parameter uncertainty $w=0.1$. 
the origin of the system (39) for all $x \in X$ by the LaSalle's invariant principle [16].

\subsection{Robustness of the input}

According to Hauser [1] and Teel [3], inputs designed by conventional dynamic extension method lose their robustness. This is because input transformation corrupt robustness of it's input generally.

In this section, we consider the robustness of the proposed adaptive control input. When the battery voltage of aircraft decrease, the torque of the thrust also decrease. It means that the control input ú showed in (40) changes drastically. We consider this situation; add the input uncertainty $\Delta$ in system (39) and consider the following system:

$$
\dot{x}=f(x)+g_{1}(x) \Delta_{1}\left(\tilde{u}_{1}+w\right)+g_{2}(x) \Delta_{2} u_{2},
$$

We use the input $u$ proposed in the Theorem 4 and asymptotically stabilizes the origin of the system (43).

Theorem 5. Consider the system (43). The control input $u$ proposed in the Theorem 4 asymptotically stabilizes the origin of the system (43) for all $x \in X, \Delta \in[1 / 2,+\infty)$ :
We show a simple proof of the Theorem 4

Proof 7. Consider the following proper positive definite function $V: X \times \mathbb{R} \rightarrow \mathbb{R}_{\geq 0}$ :

$$
V(x, \hat{w})=V(x)+\frac{1}{2} \Delta_{1}(w-\hat{w})^{2} .
$$

Here, the following equations hold:

$$
\begin{gathered}
\dot{V}(x, \hat{w})=\dot{V}(x)-\dot{\hat{w}}(w-\hat{w}) \\
=L_{f} V+L_{g_{1}} V \times \Delta_{1} k_{1}(x)+L_{g_{2}} V \times \Delta_{2} k_{2} \\
\leq 0 .
\end{gathered}
$$

Here, the above inequality is satisfied because the input $u=\left[k_{1}(x), k_{2}(x)\right]$ have the gain margin $\in[1 / 2,+\infty)$. Then the function $V$ is a weak Lyapunov function for system (39) by the LaSalle's invariant principle [16]. Therefore, the controller asymptotically stabilizes the origin of system (39) for all $x \in X, \Delta \in[1 / 2,+\infty)$.

Here, we show a simulation of the the system (39) under $w=0.1, \Delta_{1}=0.5, \Delta_{2}=0.6$ and a controller designed by Theorem 4 . State response converges to the origin in Fig. 4. Estimated value $\hat{w}$ converges to $w=0.1$. We can confirm that the controller compensates the offset error depicted in Fig. 3.

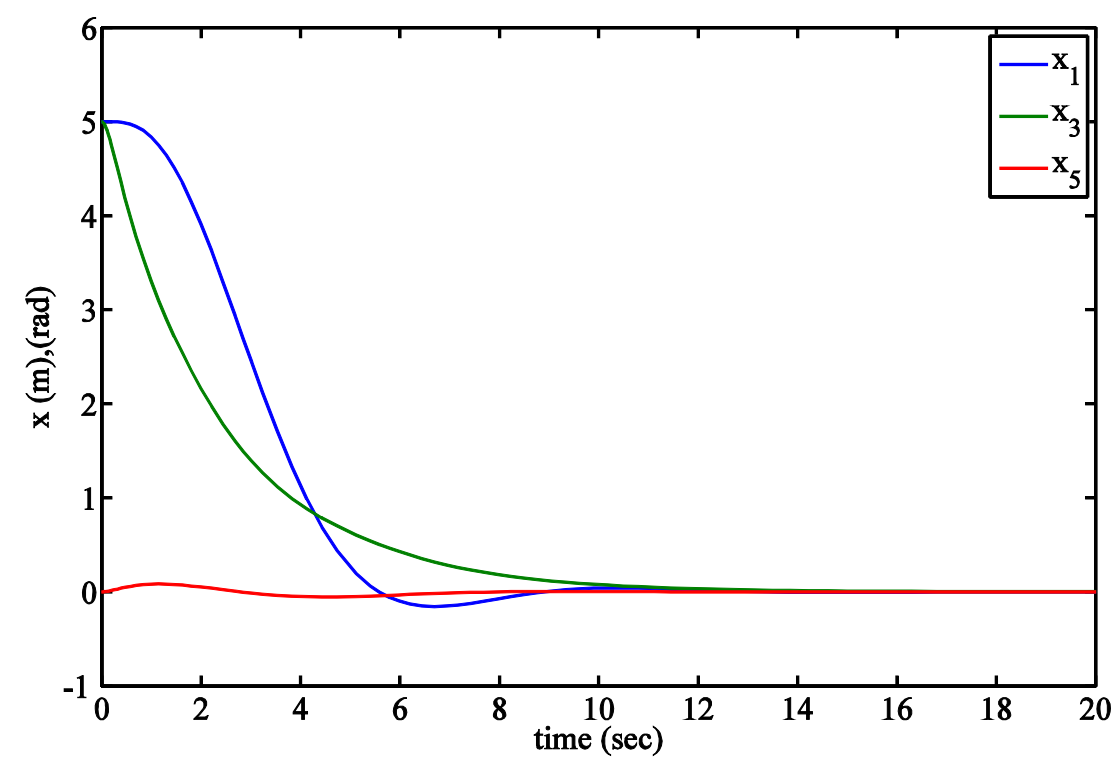

Fig. 4 State response with adaptive control $x(0)=(0.5,0,0.5,0,0,0)$ under parameter uncertainty $w=0.1$. 


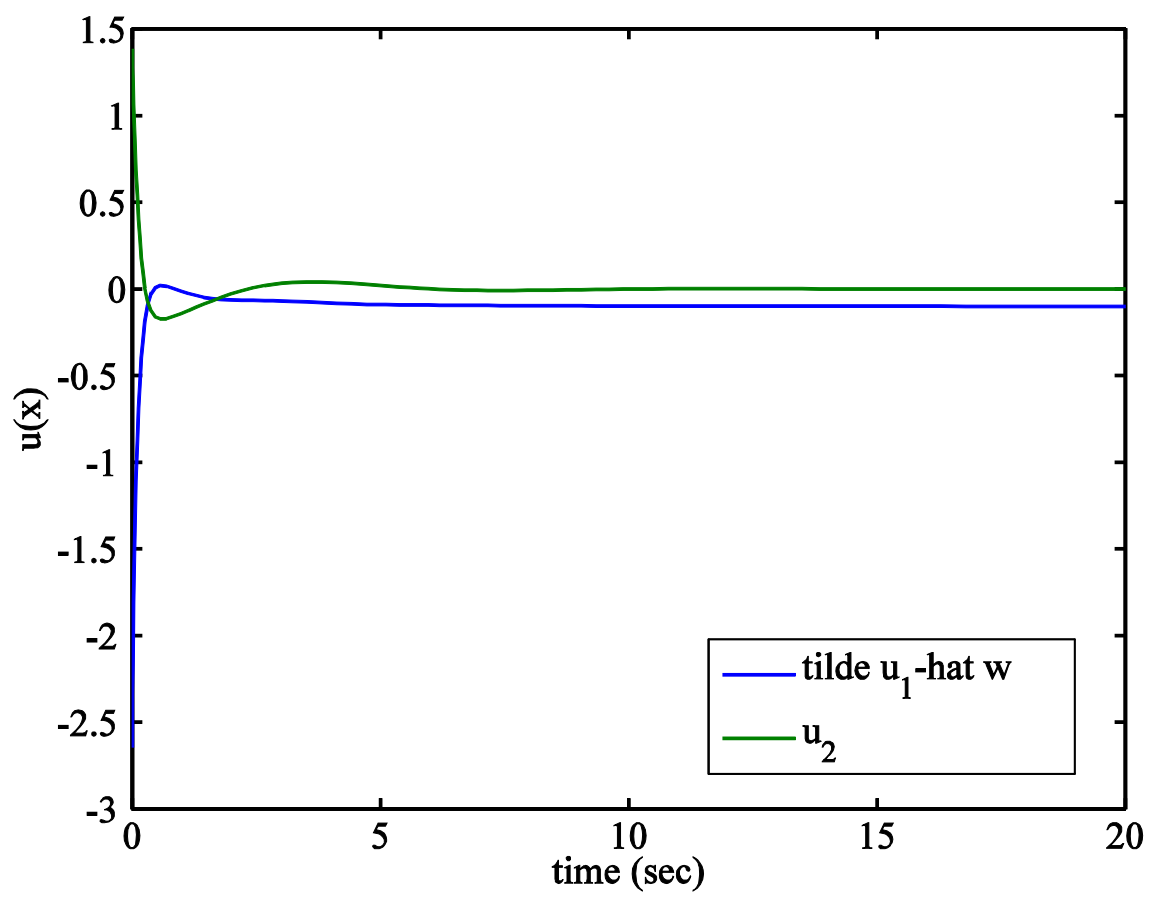

Fig. 5 Input response with adaptive control $x(0)=(0.5,0,0.5,0,0,0)$ under parameter uncertainty $w=0.1$.

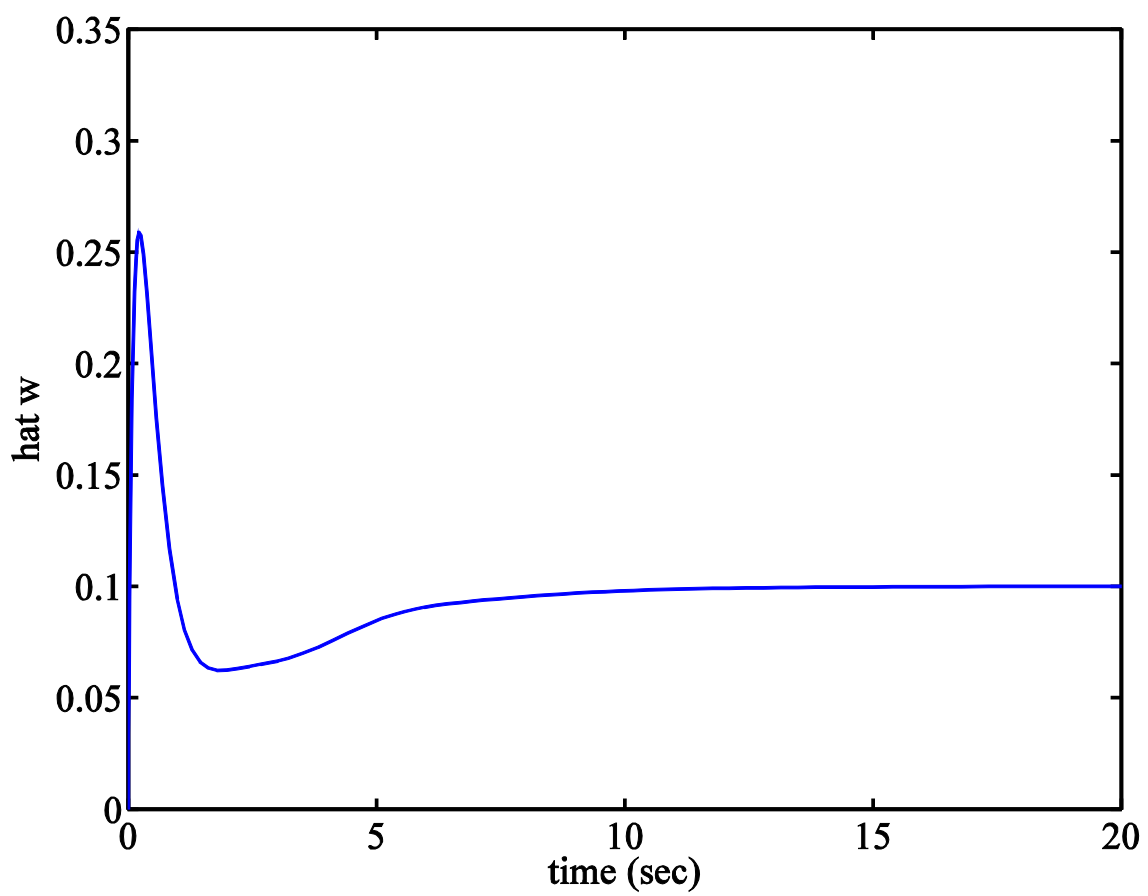

Fig. 6 Estimated value $\hat{w}, x(0)=(0.5,0,0.5,0,0,0)$ under parameter uncertainty $w=0.1$. 


\section{Conclusion}

This paper considers a static state feedback controller design problem for the PVTOL system and its robustness. We proposed smooth CLFs for the PVTOL system via minimum projection method and dynamic extension. We apply inverse optimal control and adaptivecontrol based on smooth CLF.

We confirm that the proposed controller successfully stabilizes a desired operating point.

Note that the proof Theorem 2 are not limited to the case of the PVTOL system. The results in the paper would extended to general differentially flat control systems. This remains future study.

\section{Acknowledgement}

This work was supported by JSPS KAKENHI Grant Number 15H04022.

\section{References}

[1] J. Hauser, S. Sastry and G. Meyer, Nonlinear control design for slightly nonminimum phase systems: Application to V/STOL Aircraft, Automatica, volume 28, pp. 665-679, (1992).

[2] Turker Turker, Tugce Oflaz, Haluk Gorgun and Galip Cansever, A Stabilizing Controller for PVTOL Aircraft, American Control Conference, pp. 909-913, (2012).

[3] A. R. Teel, A nonlinear small gain theorem for the analysis of control systems with saturation, IEEE Transactions on Automatic Control, volume 41, pp. 1256-1270, (1996).

[4] I. Fantoni and R. Lozano, Non-linear Control for Underactuated Mechanical Systems, Springer, (2002).

[5] O. Saber, Global configuration stabilization for VTOL aircraft with strong input coupling, IEEE Transaction on Automatic Control, volume 47, pp. 1949-1952, (2002).

[6] Minh-Uuc Hua, Tarek Hamel, Pascal Monrin and Claude Samason, Introduction to Feedback Control of Underactuated VTOL Vehicles, IEEE Control Systems Magazine, volume 33, (2013).

[7] T. Yamazaki, Y. Yamashita and H. Nakamura, Static nonsmooth control Lyapunov function design via dynamic extension, 50th IEEE Conference on Decision and Control and European Control Conference (CDC-ECC), pp. 7573-7578, (2011).

[8] Andrea Bacciotti and Lionel Rosier, Liapunov Functions and Stability in Control Theory, Springer, (2001).

[9] R. A. Freeman, P. V. Kokotovi, Robust Nonlinear Control Design: State-Space and Lyapunov Techniques, (1996).

[10] Nami Nakamura, H. Nakamura and H. Nishitani, Global inverse optimal control with guaranteed convergence rates of input affine nonlinear systems, IEEE Transactions on Automatic Control, (2011).

[11] Zvi Artstein, Stabilization with relaxed controls, Nonlinear Analysis, Theory, Methods \& Applications, volume 7, No.11, pp. 1163-1173, (1983).

[12] A. Isidori, Nonlienar Control Systems, Springer, (1995).

[13] Michel Fliess, Jean Lévine, Philippe Martin, and Pierre Rouchon, A Lie-Backlund Approach to Equivalence and Flatness of Nonlinear Systems, IEEE TRANSACTIONS ON AUTOMATIC CONTROL, volume 44, pp. 922-937, (1999).

[14] I. Fantoni, A. Zavala and R. Lonzano, Global stabilization of a PVTOL aircraft with bounded thrust, 41th IEEE Conference on Decision and Control, volume 76, pp. 1833-1844, (2002).

[15] H. Nakamura, Y. Yamashita and H. Nishitani, Minimum Projection Method for nonsmooth control Lyapunov function design on general manifolds, Systems \& and Control Letters, pp. 716-723, (2009).

[16] J. P. LaSalle, The Stability of Dynamical Systems, Siam, (1976). 